\title{
STUDY ON A SINGLE INTERPOLATION FUSION ALGORITHM FOR MULTISOURCE REMOTE SENSING DATA OF SOIL MOISTURE
}

\author{
YU, J. S. ${ }^{1}-$ CHEN, J. P. ${ }^{1 \#}-$ LI, X. J. ${ }^{2 \#}-$ LIU, Y. M. ${ }^{1 \#}-$ YAO, X. L. ${ }^{1,2^{*}}$ \\ ${ }^{I}$ College of Water Sciences, Beijing Normal University, No. 19 Xinjiekouwai Street, Beijing \\ 100875, China \\ ${ }^{2}$ College of Resource Environment and Tourism, Capital Normal University, No. 105 \\ XiSanhuan North Road, Beijing 100048, China \\ ${ }^{\#}$ These authors contributed equally to this work \\ *Corresponding author \\ e-mail: yaoxiaolei87@163.com; phone: +86-10-6225-1121 \\ (Received 22 ${ }^{\text {nd }}$ Apr 2019; accepted 12 $2^{\text {th }}$ Jul 2019)
}

\begin{abstract}
Remote sensing of soil moisture can provide important data for monitoring large-scale agricultural drought. Due to differences between the various sensors and inversion methods, remote sensing data from different sources are unsuitable for direct comparison and analysis. Data fusion has become an area of active research regarding the application of remote sensing data. Based on the principle of cumulative distribution function matching, this study proposed a continuous relationship establishment algorithm for multisource remote sensing soil moisture data. Using this new algorithm, soil Moisture and Ocean Salinity (SMOS) and Climate Change Initiative (CCI) satellite data from the Songnen Plain as test data were fused to a long time series product of real-time remote sensing soil moisture data. This application validation of this new method to SMOS and CCI indicated that this Lagrange interpolation continuous fusion algorithm could improve the fusion accuracy of multisource remote sensing soil moisture data significantly. The low-value region of the cumulative probability distribution curve is a crucial data segment for characterization of agricultural drought. Through implementation of the proposed continuous fusion algorithm, fused SMOS and CCI data were found to have high coincidence at each quantile in the low-value region of the curve.
\end{abstract}

Keywords: agricultural drought, continuous fusion, Songnen Plain, SMOS, CCI

\section{Introduction}

Remote sensing of soil moisture has been used in simulation, monitoring and analysis of drought characteristics (Atlas, 1993; Su, 2003; Sheffield and Wood, 2007; Bolten et al., 2010). It is effective for quickly detecting change in large-scale agricultural droughts. However, such remote sensing data can have different spatial and temporal resolutions and various time series lengths due to the variety of platforms, sensors and inversion methods available. Satellite data of the same terrestrial object released by different sources cannot be used directly for continuous analysis and the absolute value cannot be compared directly. To overcome this problem, remote sensing data fusion is proposed as an effective means of integrating multisource satellite-derived ground object information (Schmitt and Zhu, 2007, 2016).

Data fusion is defined as a multilevel integration process for detection, association, analysis and a combination of sensors designed to produce a single signal of higher quality and reliability (Waltz and Llinas, 1990; Sportouche et al., 2011; Li et al., 1995). Due to the resource complementarity of multiple sensors, multisource fusion can 
provide information that is more comprehensive than obtained from a single sensor (Khaleghi, 2013). The data fusion principle is the cumulative distribution function (CDF) matching method. The CDF principle was first proposed by Calheiros in 1987 (2010), and it has since been used for the correction of radar remote sensing precipitation data (Atlas et al., 1990; Anagnostou et al., 2010). In recent years, this CDF matching principle is widely used to reduce the systematic biases between the original data and the reference data by rescaling the target data to the direction of the reference data (Reichle, 2004; Drusch, 2005; Brocca et al., 2011; Lee and Im, 2015).

In the implementation of the CDF principle based on some reliable data, the fusion of remote sensing data from other sources can be used to improve the spatiotemporal resolution of the data or to extend the length of the data time series. Fused remote sensing data can improve its quality and reliability and enable its maximal utilization (Li et al., 1995). Previous studies that have used this principle to conduct fusion research on different remote sensing data groups have reported reduced remote sensing data bias and improved time series lengths for single data sources (Reichle and Koster, 2004; Liu et al., 2009). Based on this principle, the same basic data can be used to correct the fusion of multiple remote sensing datasets from different sources (Liu et al., 2010). In recent years, this principle has also been applied to the data fusion of multiple remote sensing soil moisture products (Lee et al., 2017). The CDF principle can maintain the original relative change mode of remote sensing data (Liu et al., 2010) and adjust the data scope to be close to the real value, which can improve the accuracy of remote sensing soil moisture data for characterization of surface features. However, the above calculation algorithm of fusion is simplistic and the fusion precision needs to be improved. The piecewise linear regression method is generally used to establish the relationship between the cumulative distribution curves of data from different sources. This method sets the same section for different cumulative distribution curves, and it obtains the linear equation of the two data correlations in that section to realize the fusion of one set of data to the other on the same quantile. The main disadvantage of subsection fusion is that the subsection properties will lead to a certain fitting error of the curve in the subsection, irrespective of the subsection density.

Given the increasing levels of research into remote sensing products and their application, the demands for data in terms of time series length and real-time update speed have increased. Improvement of the accuracy of multisource remote sensing data fusion is one of the most important scientific problems to be overcome in relation to research of remote sensing applications. Based on the CDF matching theory, this study constructed a new continuous fusion algorithm to address the problem of low accuracy in the piecewise linear fusion method, to improve the fusion accuracy in the low-value region of the cumulative probability distribution curve (i.e., this critical stage related to impending or emerging drought). The fusion effect of this proposed algorithm was verified by SMOS and CCI data in the Songnen Plain (Northeast China).

\section{Data and study area}

\section{Soil moisture and ocean salinity (SMOS) data}

The Soil Moisture and Ocean Salinity (SMOS) satellite designed specifically for monitoring global soil moisture content and ocean salinity. The L-band, which is insensitive to surface roughness and vegetation coverage, is very suitable for soil moisture inversion. The SMOS soil moisture data are obtained using a radiative transfer 
model using luminance temperature data (Calvet et al., 2011; Ramirez-Beltran et al., 2010). Related study has proven that the SMOS soil moisture data has high inversion accuracy and signification advantage over other remote sensing data in areas with high vegetation coverage (Al-Yaari et al., 2014). The daily SMOS Level-3 volumetric $\left(\mathrm{m}^{3} \mathrm{~m}^{-3}\right)$ soil moisture data (January 2010 to March 2016) used in this study were obtained from the expert center in Barcelona, Spain (http://www.smos-bec.icm.csic.es/smos_products). For SMOS data, radio frequency interference (RFI) is revealed to be an important source of noise (Anterrieu, 2011). This dataset discarded some data which do not meet the quality requirement in the processing of RFI. The Level-3 data in this dataset is remapped to an Equal Area Scalable Earth grid of $25 \mathrm{~km} \times 25 \mathrm{~km}$. According to the data analysis, during the main growth stage of crops from April to October, this dataset still has a high coverage in Northeast China. This daily SMOS dataset is a near realtime soil moisture data (delay one day). In the management of agricultural drought resistance, timeliness is important for the evaluation of agricultural drought. But this data cannot be used to perform frequency and evolution analysis due to the short length of the time series.

\section{Climate change initiative (CCI) data}

The European Space Agency (ESA) launched the Climate Change Initiative (CCI) program in 2010 to generate a dataset of global soil moisture based on passive, active and fused products (Dorigo et al., 2017). This program was designed to produce complete and consistent global soil moisture dataset by several active and passive remote sensing data. The long time series CCI dataset, which comprises a variety of integrated multisatellite soil moisture products, provides the possibility for long-term dynamic analysis of global soil moisture (Hollmann et al., 2013). This dataset has been widely adopted since its release, and it has been verified and applied in many regions of the world (González-Zamora et al., 2018; Jian et al., 2018; Wang et al., 2018; Siyu et al., 2017). As reprocessing data, real-time drought evaluation cannot be undertaken using this dataset. This study uses daily CCI (version 02.2) volumetric $\left(\mathrm{m}^{3} \mathrm{~m}^{-3}\right)$ combined data (1978-2014) to carry out the algorithm validation. These daily soil moisture products have a grid resolution of $0.25^{\circ}$ (Chakravorty et al., 2016).

The fusion calculation algorithm proposed in this study can extent the time series of remote sensing data. And it will have another significant advantage if the new fusion data has the property of near real-time update at the same time. Therefore, in this study CCI data is the basic data in fusion calculation. Through fusion calculation, the realtime update SMOS data has been rescaled to CCI to produce the new fusion data, which has the property of both long time series and real-time update. Before fusion, both SMOS and CCI data have been rescaled to the same spatial and temporal resolution.

\section{Study area}

This study verified the proposed continuous algorithm by application to an area of the Songnen Plain $\left(43^{\circ} 36^{\prime}-49^{\circ} 45^{\prime} \mathrm{N}, 121^{\circ} 27^{\prime}-128^{\circ} 12^{\prime} \mathrm{E}\right)$, which is one of the main bases for grain production in Northeast China (Wang et al., 2004). The Songnen Plain has a temperate semihumid-semiarid continental monsoon climate (Zheng et al., 2015). Mean annual precipitation is $350-600 \mathrm{~mm}$ with $70-80 \%$ of precipitation occurring in June-September. The typical zonal soils are black soil and chernozem. The Songnen Plain is within the drainage area of the Songhua River. The Second 
Songhua River and the Nen River merge in the center of the area to form the Songhua River. In recent years, mean precipitation on the Songnen Plain has declined and the area has suffered frequent drought disasters, especially in western regions. Due to its flat terrain, simple underlying surface type and wide distribution of farmland, this region is ideal for research on remote sensing evaluation of drought. The elevation of the Songnen Plain is $200-250 \mathrm{~m}$ above sea level. The soil in this region is fertile with vast farmland and few hills. Black soil, meadow soil, dark-brown soil and chernozem are widely distributed in there (Jiang et al., 2016).

\section{Methodology}

Data fusion in this study is to rescale one data against another basic data by the relationship obtained from data for the overlapping period. The CDF matching method is the principle of establishing relationship by cumulative distribution curves in data fusion. The calculation error of fusion in the process of establishing the relationship between two curves is very important because it determines the accuracy of the data produced for subsequent application. Currently, the relationship establish algorithm is piecewise linear regression. The relational fitting curve constructed by piecewise might lead to large error in the fusion result. To reduce error and improve fusion accuracy, this study proposed a continuous relationship establish method in fusion of multisource remote sensing soil moisture data based on the CDF principle. By using this new continuous algorithm, the overlapping period (2010-2013) data can be used to establish the relationship between CCI and SMOS in each quantile of the cumulative probability distribution curve. In the absence of CCI soil moisture data, the relationship of each quantile can rescale real-time SMOS to $\mathrm{CCI}$, consequently to produce a new fusion data with the property of long time series and real-time update.

\section{Cumulative distribution function (CDF)}

The CDF is the sum of the probabilities that a random variable falls within a certain interval in the sample space, and it is the integral of the probability density function. When plotting the integral distribution function, it is often defined as the integral of the histogram distribution because the real probability distribution function is unknown. The objective of this study is to derive an algorithm by using CDF principle to fuse real-time SMOS data to the benchmark long time series CCI remote sensing soil moisture data. This new algorithm can make the SMOS and CCI data having the similar distribution curves. Subsequently, a set of real-time updated and long-term remote sensing soil moisture data could be synthesized.

The fusion process of soil moisture data can be expressed as follows:

$$
c d f_{c}\left(x^{\prime}\right)=c d f_{s}(x)
$$

where $c d f_{c}$ is the CDF of the CCI soil moisture, $c d f_{s}$ is the CDF of the SMOS soil moisture, $x$ is the soil moisture of the SMOS product and $x$ ' is the fused SMOS soil moisture. In fusion computing, the closer the match of the two curves, the better the fusion precision and the effect. 


\section{Piecewise linear algorithm}

Piecewise linear regression is the commonly used correlation calculation method in fusion and its calculation process is shown in Figure 1. As an example, the cumulative probability distribution curves of the SMOS and CCI datasets were divided into 10 segments: $0 \%, 10 \%, 20 \%, 30 \%, 40 \%, 50 \%, 60 \%, 70 \%, 80 \%, 90 \%$ and $100 \%$. The correlation between their respective cumulative probability distribution curves can be obtained based on the linear equation of these two datasets in each segment.

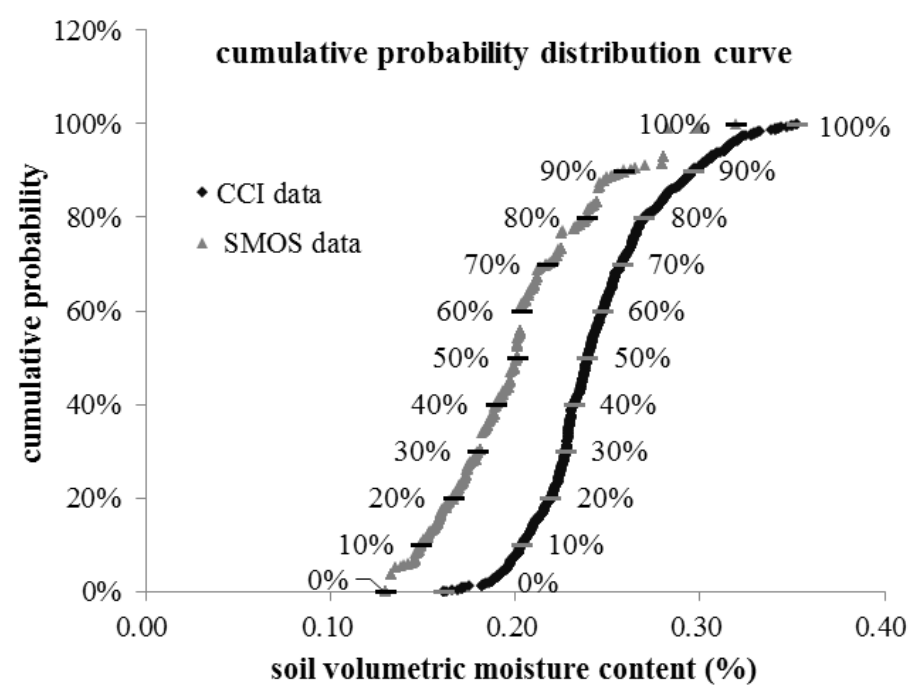

Figure 1. Schematic of piecewise linear fusion between SMOS and CCI soil moisture

\section{Lagrange continuous algorithm}

To improve fusion accuracy, correlation calculation algorithms of cumulative probability distribution curve between different data need to be further improved. This study proposed a Lagrange continuous algorithm to establish the relationship between the SMOS and CCI cumulative probability distribution curves. Between the cumulative probability distribution curves of the SMOS and CCI datasets, each SMOS value has a corresponding probability distribution value $P$. On the CCI curve, if a CCI value corresponds to this probability value $P$, this CCI value is used as the fused SMOS value. If the probability value $P$ has no corresponding CCI value, an appropriate value is calculated using an interpolation method. The Lagrange equidistant interpolation continuous algorithm can realize continuous fusion of the SMOS and CCI data in each quantile on the probability distribution curve.

Interpolation refers to the interpolation of discrete data to the continuous function, such that the continuous function curve passes through all data points. Interpolation can be used to estimate an approximation of the function at other points based on the value of the function at a finite point. Unary interpolation can calculate the approximate value at interpolation point $\mathrm{t}$ using the function when given the value at $\mathrm{n}$ equidistant nodes. The Lagrange interpolation polynomial was first published by Waring in 1779, rediscovered by Euler, and published by Lagrange in 1795 (Meyer et al., 2008; Jeffreys and Jeffreys, 1988). Lagrange interpolation polynomial can be expressed as follows: 


$$
L(x)=\sum_{j=0}^{k} y_{j} \ell_{j}(x)
$$

where, $l_{j}(x)$ is the Lagrange basic polynomial (also called the interpolation basis function), which can be expressed as

$$
\ell_{j}(x)=\prod_{i=0, i \neq j}^{k} \frac{x-x_{i}}{x_{j}-x_{i}}=\frac{\left(x-x_{0}\right)}{\left(x_{j}-x_{0}\right)} \cdots \frac{\left(x-x_{j-1}\right)}{\left(x_{j}-x_{j-1}\right)} \frac{\left(x-x_{j+1}\right)}{\left(x_{j}-x_{j+1}\right)} \cdots \frac{\left(x-x_{k}\right)}{\left(x_{j}-x_{k}\right)}
$$

Through Lagrange interpolation, each SMOS data element has a corresponding CCI value, and the CCI value obtained using this interpolation method has minimal deviation on the cumulative probability curve. Figure 2 shows the cumulative distribution curve schematic of SMOS and CCI, together with the fused SMOS (labeled SMOS' in the figure) obtained using the proposed Lagrange interpolation continuous algorithm. It can be seen that the cumulative distribution curve of the SMOS' data almost overlaps with the curve of CCI data.

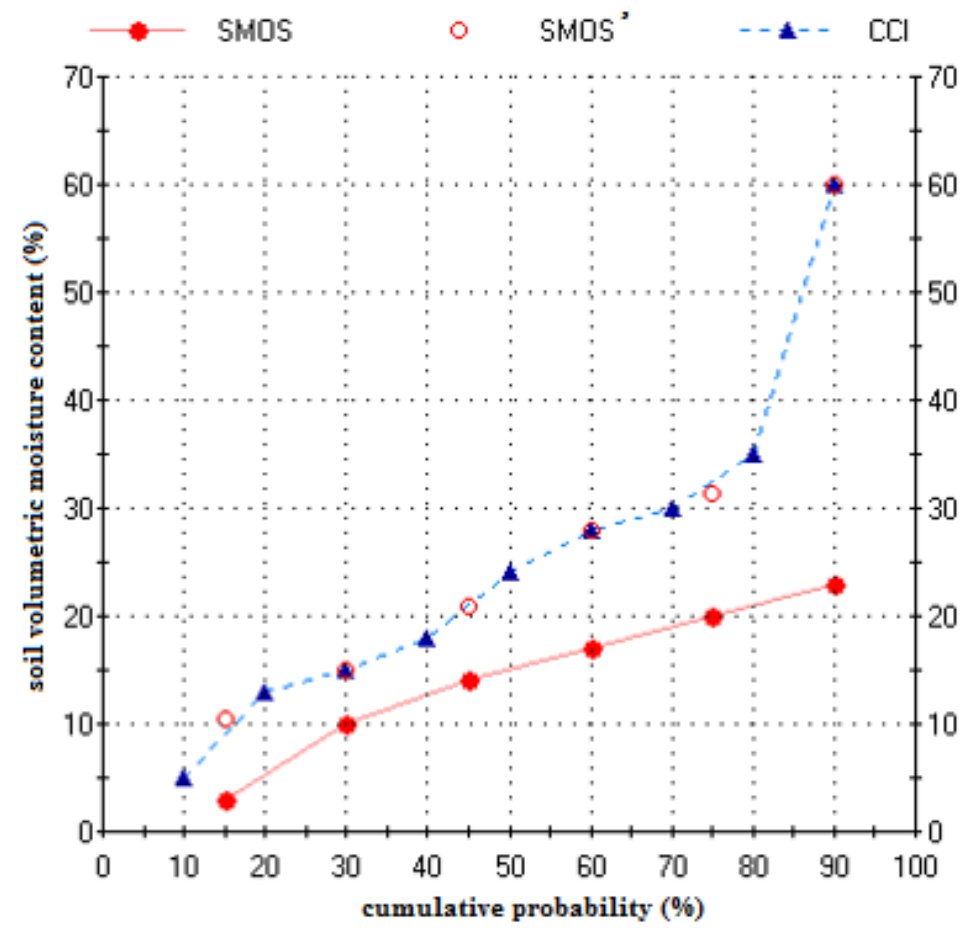

Figure 2. Schematic of Lagrange continuous fusion between SMOS and CCI soil moisture

\section{Continuous fusion algorithm results analysis}

\section{Analysis of fusion data time series}

Comparison of time series of remote sensing soil moisture data before and after fusion can illuminate the change of scope of the remote sensing data manifest by the fusion algorithm. As an example, the comparison of Shuangcheng in Songnen Plain is presented in Figure 3. 
Following the fusion process, the fused SMOS data (labeled SMOS' in Fig. 3) are clearly closer to the CCI data distribution. The values of the SMOS data time series before fusion are generally lower than the CCI data values. After fusion, the soil water content of the SMOS data is increased, especially in months with low soil water content. The SMOS' data maintain the change characteristics of the original data time series. And the range of data values is adjusted to a certain extent, enhancing the degree of similarity with the CCI data range. In the case of Shuangcheng, the SMOS data range of Shuangcheng changed from $13-30 \%$ to $19-35 \%$ through fusion.

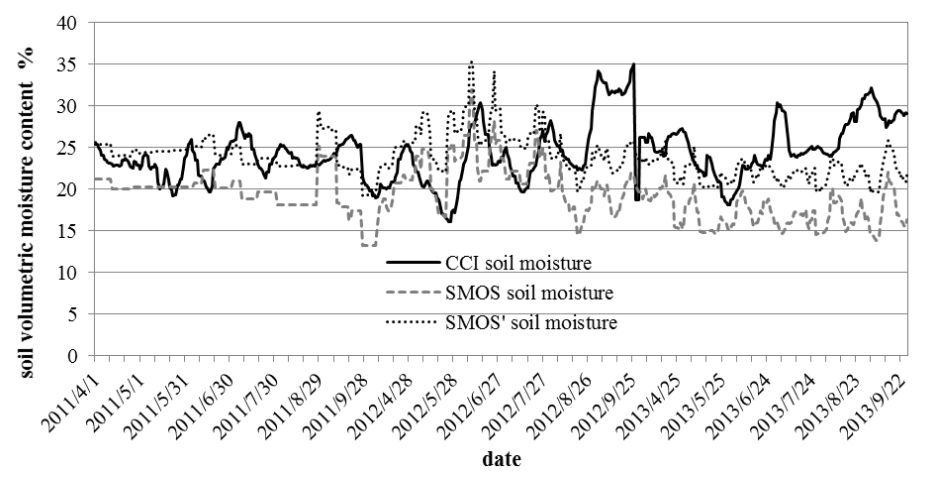

Figure 3. Soil moisture time series of SMOS, CCI and fusion SMOS data in Shuangcheng

\section{Comparison of cumulative probability distributions between the two fusion methods}

According to the CDF matching principle, the closer the curve of the target data to the cumulative probability distribution curve, the higher the fusion accuracy. Here, the result of the fusion of SMOS and CCI data of Shuangcheng (in Songnen Plain) is taken as an example for comparative analysis. The relationship between the cumulative probability distribution curves of the fused SMOS (labeled SMOS' in Fig. 3) and CCI data fused by piecewise linear algorithm and the Lagrange continuous algorithm is shown in Figure $4 a$ and $b$, respectively.

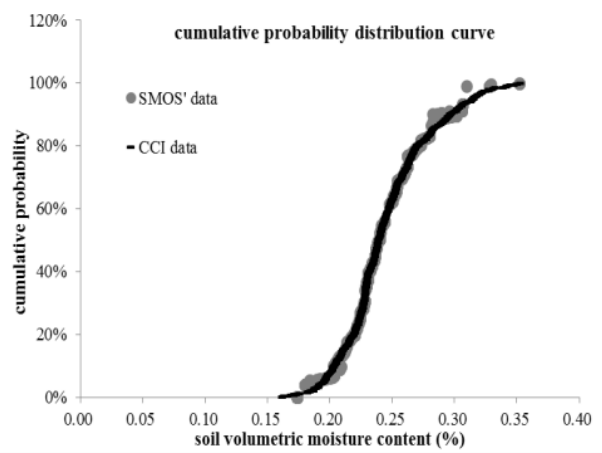

a. Piecewise linear fusion method

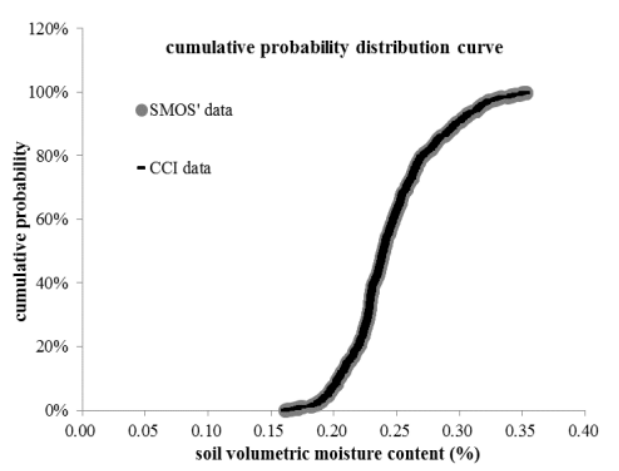

b. Lagrange interpolation fusion method

Figure 4. Cumulative probability distributions derived using (a) piecewise linear algorithm and (b) Lagrange continuous algorithm in Shuangcheng

The result of the piecewise linear fusion shows certain error in SMOS' data, especially in the low-value region that is indicative of drought. This error would cause 
loss of accuracy in real-time drought evaluation based on this fused soil moisture. The correlation established using the linear equation in each segment is the cause of this error. The result of the Lagrange continuous fusion shows the cumulative probability distribution curves of the SMOS' data (fused data) and CCI data (target data) coincide almost exactly (Fig. 4b). The two curves retain a high degree of coincidence in the lowvalue region of the curve. In terms of the cumulative probability distribution curve, the Lagrange continuous algorithm has higher fusion accuracy than the piecewise linear algorithm in fusion calculation.

The fusion precision of the two algorithms is also assessed quantitatively assessed by determining the coefficient of correlation $\left(R^{2}\right)$ and the Nash efficiency coefficient $\left(E_{N S}\right)$. The Nash efficiency coefficient can be calculated as follows:

$$
E_{n s}=1-\frac{\sum_{i=1}^{n}\left(O_{i}-P_{i}\right)^{2}}{\sum_{i=1}^{n}\left(O_{i}-\bar{O}\right)^{2}}
$$

where $O_{i}$ and $P_{i}$ represent the CCI and SMOS' data, respectively, and $\bar{O}$ is the average of the CCI data. The closer the value of $E_{N S}$ to 1, the better the degree of coincidence between the two results.

The values of $R^{2}$ and $E_{N S}$ of the two curves obtained using the piecewise linear algorithm were 0.98 and 0.97 , respectively. And the $R^{2}$ and $E_{N S}$ values in the low-value region were 0.73 and 0.52 , respectively. The values of $R^{2}$ and $E_{N S}$ of the two curves obtained using the Lagrange continuous algorithm were 0.99 and 0.99 , respectively. And the $R^{2}$ and $E_{N S}$ values in the low-value region were also 0.99 and 0.99 , respectively. Therefore, it is evident that the Lagrange continuous algorithm improves the fusion precision significantly in the low-value region which be used for the characterization of drought.

\section{Verification of remote sensing soil moisture by drought event}

The fused SMOS data (SMOS' data), SMOS and CCI data were also evaluated for accuracy of drought expression. Site observation data from six stations within the study area were used as the basis for quantitative evaluation of the degree of consistency between the remote sensing data and the site observation data in the expression of drought events. The in situ data comes from China crop growth and development data set in China meteorological data network. This dataset contains moisture data per tenday for 7 soil depths at each site. The surface $10 \mathrm{~cm}$ data was compared with the remote sensing data for verification. Here, as quantitative evaluation indices, hit rate $(H)$, false alarm rate $(F)$ and equitable threat score $(E T S)$ are used to reflect the level of agreement of remote sensing of soil moisture with drought events (Grayson and Western, 1998). The higher the ETS value, higher the $H$ value and lower the $F$ value, the closer the level of agreement between the remote sensing data and drought events. The cumulative probability distribution of $30 \%$ is used as the threshold of the occurrence of drought both for remote sensing data and in situ data.

These evaluation indices define $a$ as the number of events when drought is shown to occur in both the in situ observations and the fused SMOS data, $b$ is when drought occurs in the fused SMOS data but not in the in situ observations, $c$ is when drought occurs in the in situ observations but not in the fused SMOS data and $d$ is when drought 
does not occur in either the fused SMOS data or the in situ observations. Then, $H$ can be expressed as follows:

$$
H=\frac{a}{a+b}
$$

where the range of values of $H$ is $0-1$ ( 1 being the best); $F$ can be written as

$$
F=\frac{b}{b+d}
$$

where the range of values of $F$ is $0-1$ ( 0 being the best) and ETS can be expressed as

$$
E T S=\frac{a-a_{r e f}}{a-a_{r e f}+b+c}
$$

where the range of values of ETS is $-1 / 3$ to 1 ( 1 being the best). Here, $a_{r e f}$ is expressed as

$$
a_{r e f}=\frac{(a+b)(a+c)}{(a+b+c+d)}
$$

The drought verification of SMOS, CCI and SMOS' data for the six sites in the study area is shown in Table 1. CCI (version 02.2) dataset produced by several active and passive remote sensing data has integrally highest hit rate and equitable threat score. SMOS data as a single data source has integrally lowest hit rate and equitable threat score. SMOS' data is a combination of CCI and SMOS, with real-time update feature as with SMOS, and has significantly more accurate results for drought events verification than SMOS. The fused SMOS data has the characteristics of long time series and realtime update, which achieves the purpose of fusion research in this paper. In terms of drought event validation, SMOS' has a better validation effect than SMOS, indicating that this SMOS' dataset can be applied to drought evaluation research.

Table 1. Drought verification by in situ data

\begin{tabular}{c|c|c|c|c|c|c|c|c|c}
\hline \multirow{2}{*}{ Site } & \multicolumn{3}{|c|}{ Hit rate } & \multicolumn{3}{c|}{ False alarm } & \multicolumn{3}{c}{$\begin{array}{c}\text { Equitable threat score } \\
\text { ETS }\left(\mathbf{1 0}^{-2}\right)\end{array}$} \\
\cline { 2 - 10 } & SMOS & CCI & SMOS' & SMOS & CCI & SMOS' & SMOS & CCI & SMOS' \\
\hline Keshan & 0.31 & 0.69 & 0.38 & 0.27 & 0.14 & 0.26 & 2.26 & 38.16 & 6.00 \\
Fuyu & 0.11 & 0.64 & 0.69 & 0.37 & 0.16 & 0.13 & -11.28 & 31.08 & 38.82 \\
Hailun & 0.58 & 0.33 & 0.53 & 0.18 & 0.27 & 0.21 & 25.09 & 3.26 & 19.16 \\
Tailai & 0.33 & 0.57 & 0.43 & 0.07 & 0.19 & 0.23 & 18.6 & 23 & 11.11 \\
Anda & 0.4 & 0.57 & 0.35 & 0.24 & 0.2 & 0.27 & 8.41 & 20.91 & 4.42 \\
Changling & 0.35 & 0.5 & 0.25 & 0.28 & 0.22 & 0.31 & 3.67 & 15.88 & -2.93 \\
Meihekou & 0.18 & 0.55 & 0.36 & 0.33 & 0.19 & 0.28 & -7.04 & 21.97 & 4.36 \\
\hline
\end{tabular}




\section{Comparison of CCI data between version 02.2 and version 04.2}

The latest version of CCI soil moisture product (v04.2) has already included the SMOS soil moisture. Figure 5 shows the time series comparison of two different version of CCI data in a county in the study area. The new version of CCI data that incorporates SMOS is more similar in scope to SMOS data. The time series of CCI (v04.2) combined dataset is form 1978 to 2016, and the timeliness limited the application in real-time evaluation. The fusion method proposed in this paper can generate near-real-time, long-sequence data products that can reflect real drought events more accurately than SMOS.

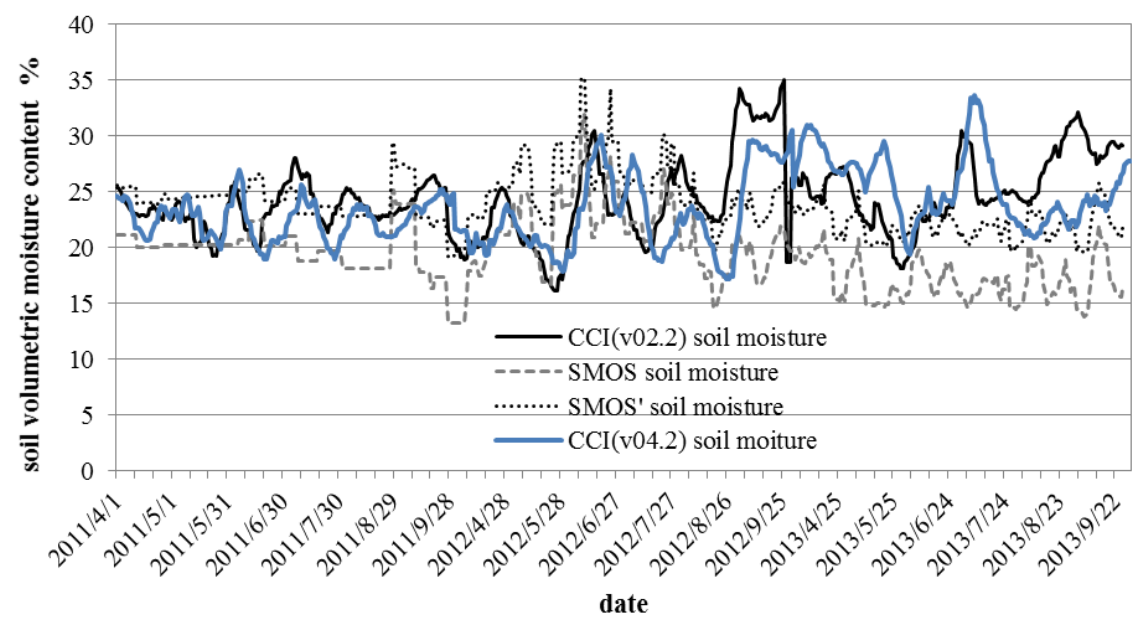

Figure 5. Soil moisture time series of CCI(v02.2), CCI(v04.2), SMOS, and SMOS' data in Shuangcheng

\section{Discussion and conclusion}

The efficacy of the application of remote sensing soil moisture data to the evaluation of agricultural drought is reflected mainly in the validity and accuracy of the remote sensing data when soil moisture is low (i.e., impending or emerging drought). Remote sensing data fusion technology that uses one dataset to extend a time series or to improve the update speed of another dataset is an important method with which to improve the performance of remote sensing data application. Fusion precision and the fusion algorithm are the primary problems associated with the application of such techniques to remote sensing datasets.

The existing piecewise linear fusion method can produce certain deviation, especially in the low-value region of a soil moisture curve that represents the stage of impending or emerging drought. Such deviation could easily lead to large errors in fused data used in drought forecasting, which could result in the misreporting or omission of agricultural drought events. Adopting the fusion principle of cumulative distribution function matching, this study constructed a continuous fusion algorithm, based on which analysis of the precision of the fusion of Soil Moisture and Ocean Salinity (SMOS) satellite data with Climate Change Initiative (CCI) satellite data was conducted at county level on the Songnen Plain (Northeast China). It was demonstrated that the proposed Lagrange continuous fusion algorithm could improve the fusion precision of 
multisource remote sensing data and enhance the sensitivity of remote sensing soil moisture data in the characterization of drought.

Through application of the fusion algorithm, a fused CCI-SMOS remote sensing soil water product was generated at the county level for the Songnen Plain area. Through analysis of time series data before and after the fusion process, it was revealed that the fused SMOS data range was closer to the CCI data range than the original data, while the relative change mode of the original data was retained. After the in situ data verification, fused SMOS data has a better validation effect than SMOS. The systematic deviation of the fused remote sensing soil water product was reduced, while displaying the advantages of a long time sequence and near real-time features. The results of this study could provide data support for further real-time evaluation and frequency analysis of large-scale agricultural drought on the regional scale. Validation analysis studies between the other different data sources and in other regions are needed to further verify the availability of this new fusion method.

Acknowledgments. This study was supported by the National Natural Science Foundation of China (Grant No. 51779007, 41671018), National Key Research and Development Program of China (Grant No. 2016YFC0401308), and Project funded by China Postdoctoral Science Foundation (2018M631707).

\section{REFERENCES}

[1] Al-Yaari, A., Wigneron, J. P., Ducharne, A., et al. (2014): Global-scale comparison of passive (SMOS) and active (ASCAT) satellite based microwave soil moisture retrievals with soil moisture simulations (MERRA-Land). - Remote Sensing of Environment 152: 614-626.

[2] Anagnostou, E. N., Negri, A. J., Adler, R. F. (2010): Statistical adjustment of satellite microwave monthly rainfall estimates over Amazonia. - Journal of Applied Meteorology 38(11): 1590-1598.

[3] Anterrieu, E. (2011): On the detection and quantification of RFI in L1a signals provided by SMOS. - IEEE Transactions on Geoscience and Remote Sensing 49(10): 3986-3992.

[4] Atlas, D., Rosenfeld, D., Wolff, D. B. (1990): Climatologically tuned reflectivity-rain rate relations and links to area-time integrals. - Journal of Applied Meteorology 29(11): 11201135 .

[5] Atlas, R., Woldson, N., Terry, J. (1993): The effect of SST and soil moisture anomalies on GLA model simulations of the 1988 U.S. summer drought. - J. Clim. 6: 2034-2048.

[6] Bolten, J. D., Crow, W. T., Zhan, X., et al. (2010): Evaluating the Utility of Remotely Sensed Soil Moisture Retrievals for Operational Agricultural Drought Monitoring. IEEE Journal of Selected Topics in Applied Earth Observations \& Remote Sensing 3(1): 57-66.

[7] Brocca, L., Hasenauer, S., Lacava, T., et al. (2011): Soil moisture estimation through ASCAT and AMSR-E sensors: An intercomparison and validation study across Europe. Remote Sensing of Environment 115(12): 3390-3408.

[8] Calheiros, R. V., Zawadzki, I. (2010): Reflectivity-rain rate relationships for radar hydrology in Brazil. - Journal of Applied Meteorology 26(1): 118-132.

[9] Calvet, J. C., Wigneron, J. P., Walker, J., et al. (2011): Sensitivity of passive microwave observations to soil moisture and vegetation water content: L-band to W-band. - IEEE Transactions on Geoscience \& Remote Sensing 49(4): 1190-1199.

[10] Chakravorty, A., Chahar, B. R., Sharma, O. P., et al. (2016): A regional scale performance evaluation of SMOS and ESA-CCI soil moisture products over India with 
simulated soil moisture from MERRA-Land. - Remote Sensing of Environment 186: 514-527.

[11] Dorigo, W., Wagner, W., Albergel, C., et al. (2017): ESA CCI soil moisture for improved Earth system understanding: state-of-the art and future directions. - Remote Sensing of Environment S0034425717303061.

[12] Drusch, M. (2005): Observation operators for the direct assimilation of TRMM microwave imager retrieved soil moisture. - Geophysical Research Letters 32(15):L15403.

[13] González-Zamora, Á., Sánchez, N., Pablos, M., et al. (2018): CCI soil moisture assessment with SMOS soil moisture and, in situ, data under different environmental conditions and spatial scales in Spain. - Remote Sensing of Environment S0034425718300166.

[14] Grayson, R. B., Western, A. W. (1998): Towards areal estimation of soil water content from point measurements: time and space stability of mean response. - Journal of Hydrology 207(1-2): 68-82.

[15] Sportouche, H., Tupin, F., Denise, L. (2011): Extraction and three-dimensional reconstruction of isolated buildings in urban scenes from high-resolution optical and SAR spaceborne images. - IEEE Transactions on Geoscience and Remote Sensing 49(10): 3932-3946.

[16] Hollmann, R., Merchant, C., Saunders, R., et al. (2013): The ESA Climate Change Initiative: satellite data records for essential climate variables. - Bulletin of the American Meteorological Society 94(10): 1541-1552.

[17] Jeffreys, H., Jeffreys, B. S. (1988): Lagrange's Interpolation Formula. - In: Jeffrey, H., Jeffreys, B. S. Methods of Mathematical Physics. Third Ed. - Cambridge University Press, Cambridge.

[18] Jian, K., Rui, J., Xin, L., et al. (2018): Spatial upscaling of sparse soil moisture observations based on ridge regression. - Remote Sensing 10(2): 192-202.

[19] Khaleghi, B., Khamis, A., Karray, F. O., et al. (2013): Multisensor data fusion: a review of the state-of-the-art. - Information Fusion 14(1): 28-44.

[20] Lanqi, J., Lijuan, Z., Shuying, Z., et al. (2016): Accuracy assessment of approaches to spatially explicit reconstruction of historical cropland in Songnen Plain, Northeast China. - Journal of Geographical Sciences 26(2): 219-229.

[21] Lee, C. S., Park, J. D., Shin, J., et al. (2017): Improvement of AMSR2 soil moisture products over South Korea. - IEEE Journal of Selected Topics in Applied Earth Observations \& Remote Sensing 99: 1-11.

[22] Lee, J. H., Im, J. A. (2015): Novel bias correction method for soil moisture and ocean salinity (SMOS) soil moisture: retrieval ensembles. - Remote Sensing 7(12): 1604516061.

[23] Li, N. H., Manjunath, B. S., Mitra, S. K. (1995): Multisensor image fusion using the wavelet transform. - Graphical Model and Image Processing 57(3): 235-245.

[24] Liu, Y. Y., Parinussa, R. M., Dorigo, W. A., et al. (2010): Developing an improved soil moisture dataset by blending passive and active microwave satellite-based retrievals. Hydrology \& Earth System Sciences Discussions 15(2): 425-436.

[25] Liu, Y. Y., Van Dijk, A. I. J. M., De Jeu, R. A. M., et al. (2009): An analysis of spatiotemporal variations of soil and vegetation moisture from a 29-year satellite-derived data set over mainland Australia. - Water Resources Research 45(7): 4542-4548.

[26] Meyer, R., Bo, C., Perron, F. (2008): Adaptive rejection Metropolis sampling using Lagrange interpolation polynomials of degree 2. - Computational Statistics \& Data Analysis 52(7): 3408-3423.

[27] Ramirez-Beltran, N. D., Calderon-Arteaga, C., Harmsen, E., et al. (2010): An algorithm to estimate soil moisture over vegetated areas based on in situ and remote sensing information. - International Journal of Remote Sensing 31(10): 2655-2679. 
[28] Reichle, R. H., Koster, R. D. (2004): Bias reduction in short records of satellite soil moisture. - Geophysical Research Letter 31(19): 187-206.

[29] Schmitt, M., Zhu, X. X. (2016): Data Fusion and Remote Sensing: an ever-growing relationship. - IEEE Geoscience and Remote Sensing Magazine 4(4): 6-23.

[30] Sheffield, J., Wood, E. F. (2007): Characteristics of global and regional drought, 19502000: Analysis of soil moisture data from off-line simulation of the terrestrial hydrologic cycle. - Journal of Geophysical Research 112(D17):D17115.

[31] Siyu, M., Qianxin, W., Jie, W., et al. (2017): Temporal evolution of regional drought detected from GRACE TWSA and CCI SM in Yunnan Province, China. - Remote Sensing 9(11): 1124-1138.

[32] Su, Z. (2003): Assessing relative soil moisture with remote sensing data: theory, experimental validation, and application to drought monitoring over the North China Plain. - Physics \& Chemistry of the Earth 28(1): 89-101.

[33] Waltz, E., Llinas, J. (1990): Multisensor Data Fusion. - Artech House Inc., Norwood, MA, pp. 245-253.

[34] Wang, H. X., Wan, Z. J., Yu, S. P., et al. (2004): Catastrophic eco-environmental change in the Songnen Plain, northeastern China since 1900s. - Chinese Geographical Science 14(2): 179-185.

[35] Wang, S., Mo, X., Hu, S., et al. (2018): Assessment of droughts and wheat yield loss on the North China Plain with an aggregate drought index (ADI) approach. - Ecological Indicators 87: 107-116.

[36] Zheng, S. H., Qin, Z. H., Zhang, W. B. (2015): Drought variation in Songnen Plain and its response to climate change. - Chinese Journal of Agrometeorology 36(5): 640-649. 